Standard clocks, orbital precession and the cosmological constant

This article has been downloaded from IOPscience. Please scroll down to see the full text article.

2003 Class. Quantum Grav. 202727

(http://iopscience.iop.org/0264-9381/20/13/320)

View the table of contents for this issue, or go to the journal homepage for more

Download details:

IP Address: 128.206.162.204

The article was downloaded on 20/09/2010 at 19:17

Please note that terms and conditions apply. 


\title{
Standard clocks, orbital precession and the cosmological constant
}

\author{
Andrew W Kerr, John C Hauck and Bahram Mashhoon \\ Department of Physics and Astronomy, University of Missouri-Columbia, Columbia, \\ MO 65211, USA
}

Received 7 January 2003

Published 6 June 2003

Online at stacks.iop.org/CQG/20/2727

\begin{abstract}
We discuss the influence of the cosmological constant on the gravitomagnetic clock effect and the gravitational time delay of electromagnetic rays. Moreover, we consider the relative motion of a binary system to linear order in the cosmological constant $\Lambda$. The general expression for the effect of $\Lambda$ on pericentre precession is given for arbitrary orbital eccentricity.
\end{abstract}

PACS numbers: 04.20.- q, 98.80.-k

\section{Introduction}

Current cosmological models that take the acceleration of the universe into account involve a cosmological constant $\Lambda$ of magnitude $\Lambda \approx H_{0}^{2} / c^{2}$, where $H_{0}$ is the Hubble parameter. Taking $H_{0} \approx 70 \mathrm{~km} \mathrm{~s}^{-1} \mathrm{Mpc}^{-1}$, we find that $\Lambda \approx 10^{-56} \mathrm{~cm}^{-2}$. The local effects of such a cosmological constant are expected to be very small. Nevertheless, great strides are being made in observational techniques in astronomy and it appears worthwhile to investigate some of the observational consequences of the existence of a cosmological constant.

The dependence of the gravitomagnetic clock effect on the cosmological constant is examined in section 2. Section 3 is devoted to the gravitational time delay and its dependence on $\Lambda$. The influence of the cosmological constant on the motion of a binary system is investigated in detail in sections 4 and 5. A bound orbit is shown to precess with a frequency given by $\frac{1}{2}\left(\Lambda c^{2} / \omega\right) \sqrt{1-e^{2}}$, where $\omega$ and $e$ are the orbital frequency and eccentricity, respectively. Section 6 contains a brief discussion of our results.

\section{Clock effects in the Kerr-de Sitter spacetime}

The gravitomagnetic clock effect is caused by the net spin of a gravitational source [1-4]. In general, the rotation of a massive body introduces a difference in the co- and counter-rotating orbital periods of test masses in motion around the central source. We wish to investigate this gravitomagnetic effect in the equatorial plane of the Kerr-de Sitter spacetime to find what 
influence the cosmological constant would have on the clock effect. The Kerr-de Sitter metric in the standard Boyer-Lindquist coordinates $x^{\mu}=(t, r, \theta, \phi)$ is given by $[5,6]$

$$
\begin{array}{r}
\mathrm{d} s^{2}=-\left[1-\frac{2 M r}{\Sigma}-\frac{\Lambda}{3}\left(r^{2}+a^{2} \sin ^{2} \theta\right)\right] \mathrm{d} t^{2}-2 a\left[\frac{2 M r}{\Sigma}+\frac{\Lambda}{3}\left(r^{2}+a^{2}\right)\right] \sin ^{2} \theta \mathrm{d} t \mathrm{~d} \phi \\
+\frac{\Sigma}{\Delta} \mathrm{d} r^{2}+\frac{\Sigma}{\chi} \mathrm{d} \theta^{2}+\left[\frac{2 M r}{\Sigma} a^{2} \sin ^{2} \theta+\left(1+\frac{\Lambda}{3} a^{2}\right)\left(r^{2}+a^{2}\right)\right] \sin ^{2} \theta \mathrm{d} \phi^{2},
\end{array}
$$

where units are chosen such that $G=c=1$, unless specified otherwise, and

$$
\begin{aligned}
& \Sigma=r^{2}+a^{2} \cos ^{2} \theta, \quad \chi=1+\frac{\Lambda}{3} a^{2} \cos ^{2} \theta, \\
& \Delta=r^{2}-2 M r+a^{2}-\frac{\Lambda}{3} r^{2}\left(r^{2}+a^{2}\right) .
\end{aligned}
$$

The mass of the source is $M, J=M a$ is its angular momentum and $\Lambda$ is the cosmological constant. Metric (1) represents a rotating Kottler spacetime [7]. The Kottler spacetime is also known as the Schwarzschild-de Sitter spacetime. Useful background information on de Sitter spacetime and the cosmological constant can be found in [8] and $[9,10]$, respectively.

It is interesting to note that in the Newtonian limit the physical content of equation (1) reduces to a Newtonian gravitational potential $\Phi_{N}=-G M r^{-1}-\frac{1}{6} \Lambda c^{2} r^{2}$, which satisfies the Poisson equation $\nabla^{2} \Phi_{N}=4 \pi G \rho_{N}$ with $\rho_{N}=M \delta(\mathbf{r})+\rho_{\Lambda}$. Here $\rho_{\Lambda}=-c^{2} \Lambda /(4 \pi G)$ is the effective negative uniform density of 'matter' represented by the cosmological constant. The effective repulsive force due to this source is then given by $\frac{1}{3} \Lambda c^{2} \mathbf{r}$ per unit mass. On the other hand, the relativistic interpretation of the cosmological constant in terms of vacuum energy involves a 'substance' with equation of state $\hat{\rho} c^{2}+\hat{p}=0$ such that the density $\hat{\rho}$ is positive, but the pressure is negative and is given by $\hat{p}=-c^{4} \Lambda /(8 \pi G)$.

Let us now consider circular geodesics of this spacetime. The stability of circular geodesic orbits in the equatorial plane of Kerr-de Sitter geometry has been investigated by Howes [11]. It turns out that stable circular orbits exist out to a radius of $(3 M / \Lambda)^{1 / 3}$. On the other hand, the inner stability limits vary depending on the sense of orbital motion as well as the values of $a$ and $\Lambda$ [11]. For $\Lambda=0$, we recall that for the co-rotating case, the inner stability threshold varies from $r=6 M$ to $r=M$ as $a: 0 \rightarrow M$, whereas it varies from $r=6 M$ to $r=9 M$ in the counter-rotating case.

The geodesic equation for the radial coordinate reduces to $[12,13]$

$$
g_{t t, r}\left(\frac{\mathrm{d} t}{\mathrm{~d} \phi}\right)^{2}+2 g_{t \phi, r}\left(\frac{\mathrm{d} t}{\mathrm{~d} \phi}\right)+g_{\phi \phi, r}=0,
$$

which has the solution

$$
\frac{\mathrm{d} t}{\mathrm{~d} \phi}=a \pm \omega_{K}^{-1} .
$$

Here $\omega_{K}$ is the modified Kepler frequency

$$
\omega_{K}^{2}=\frac{M}{r^{3}}-\frac{1}{3} \Lambda
$$

which can be interpreted as being due to a net mass of $M+(4 \pi / 3) \rho_{\Lambda} r^{3}$, where $\rho_{\Lambda}=-\Lambda / 4 \pi$ is the constant Newtonian density mentioned before. In connection with equation (6) as well as other modifications of Kepler's third law to include dark matter, etc, we note that the motion of planets in the solar system can be used to study deviations from Keplerian motion; however, for $\Lambda \approx 10^{-56} \mathrm{~cm}^{-2}$ the corresponding effects are too small to be detectable at present. 
There are three distinct clock effects that can be studied using counter-revolving circular geodesic orbits. To find the influence of the cosmological constant on the clock effects, we consider these in turn. Integration of equation (5) over $2 \pi$ for co-rotating and $-2 \pi$ for counter-rotating test particles implies that $t_{ \pm}=T_{K} \pm 2 \pi a$, where $T_{K}=2 \pi / \omega_{K}$ is the Keplerian period. Thus $t_{+}-t_{-}=4 \pi a$, just as in the Kerr case. Consider now an (accelerated) standard clock at rest on the circle of radius $r$ in the equatorial plane. A standard clock measures the proper time along its worldline. According to this clock, the difference in the time $\tau^{\prime}$ that it would take two free counter-revolving test particles to complete the orbit is

$$
\tau_{+}^{\prime}-\tau_{-}^{\prime}=4 \pi a \sqrt{1-\frac{2 M}{r}-\frac{\Lambda}{3}\left(r^{2}+a^{2}\right)},
$$

which is the observer-dependent single-clock clock effect according to the terminology of [12]. For the cosmological constant to have any significant influence on this clock effect, the orbital radius would have to be unreasonably large. In fact, $\Lambda \approx 10^{-56} \mathrm{~cm}^{-2}$ has a totally negligible effect even at a planetary radius of $r \approx 10 \mathrm{AU}$, since $\Lambda r^{2} / 3 \approx 10^{-28}$.

Consider now the proper periods of revolution $\tau_{ \pm}$of the two free standard clocks counterrevolving on the circular orbit of radius $r$; we find from equations (1) and (5) that

$$
\tau_{ \pm}=T_{K} \sqrt{1-\frac{3 M}{r}-\frac{\Lambda}{3} a^{2} \pm 2 a \omega_{K}} .
$$

Expanding this expression in powers of $\epsilon=a / M$, we find that

$$
\tau_{+}-\tau_{-}=\frac{4 \pi a}{\sqrt{1-\frac{3 M}{r}}}\left[1+\frac{M a^{2}\left(1-\Lambda r^{2}\right)}{2 r(r-3 M)^{2}}+O\left(\epsilon^{4}\right)\right] .
$$

Thus the cosmological constant does not affect the observer-dependent two-clock clock effect to first order in $a / M$.

Imagine now two standard clocks starting at the event characterized by $(0, r, \pi / 2,0)$, i.e. $t=0$ at $\phi=0$, and moving freely in opposite directions on the circle of radius $r$. Since the co-rotating clock is slower, their first meeting occurs at $\phi_{1}=\pi-\alpha$, where $\alpha=a \omega_{K}$. Let their $n$th meeting point be the event characterized by $t_{n}$ and $\phi_{n}$, where $t_{n}=\frac{1}{2} n T_{K}\left(1-\alpha^{2}\right)$ and $\phi_{n}=n \pi(1-\alpha)$ modulo $2 \pi$. The observer-independent two-clock clock effect refers to the difference in the proper times $\tau_{n}^{+}-\tau_{n}^{-}$of the clocks at their $n$th meeting, where

$$
\tau_{n}^{ \pm}=\frac{1}{2} n T_{K}(1 \mp \alpha) \sqrt{1-\frac{3 M}{r}-\frac{\Lambda}{3} a^{2} \pm 2 \alpha} .
$$

To first order in $a / M$, this clock effect is also independent of $\Lambda$ and is given by

$$
\tau_{n}^{+}-\tau_{n}^{-} \approx \frac{6 \pi n J}{\sqrt{r(r-3 M)}}
$$

The diametrical line joining the points of encounter with the origin of the circular orbit undergoes a precession in the opposite sense as the rotation of the source. The precession frequency is given approximately by $n \pi \alpha / \tau_{n}^{+}$, which to lowest order coincides with the precession frequency of a fixed torque-free test gyroscope in the equatorial plane at radius $r$.

The gravitomagnetic clock effect (9) far from the source, $3 M \ll r \ll(3 M / \Lambda)^{1 / 3}$, is essentially independent of $\Lambda$, and is basically proportional only to $a=J / M$, which is the specific angular momentum of the source. That is, the effect has a topological character as it is practically independent of the radius of the orbit. It is also essentially independent of the gravitational constant $G$; therefore, the effect can be 'large'. For instance, for spaceborne clocks around the Earth the effect is $\sim 10^{-7} \mathrm{~s}$; however, the detection of the effect requires knowledge of satellite orbits to millimetre accuracy [3]. This seems to be somewhat 
beyond present capabilities by about an order of magnitude; moreover, the influence of the cosmological constant on the clock effect is too small to be detectable in the foreseeable future. Another aspect of the clock effect is that it takes longer to complete a prograde circular orbit in the equatorial plane than the corresponding retrograde orbit. Thus free motion in the same sense as the rotation of the source is slower than motion in the opposite sense, which is contrary to what one might expect from the 'dragging of space' by the rotating source.

There is a close relationship between the clock effect and circular holonomy. In fact, circular holonomy in the Kerr-de Sitter spacetime can be developed in close analogy with that in the Kerr spacetime [14]. This would involve the parallel transport of a vector around a constant-time circular $\phi$-loop in the equatorial plane of the Kerr-de Sitter spacetime. Let us just mention here the result that there is a band of holonomy invariance for $r_{*}<r<(3 M / \Lambda)^{1 / 3}$ given by $\tilde{n} f(r)=\tilde{m}$, where $\tilde{n}$ and $\tilde{m}$ are positive integers and

$$
f^{2}(r)=1-\frac{2 M}{r}-\frac{2 M a^{2}}{r^{3}}-\frac{M^{2} a^{2}}{r^{4}}-\frac{1}{3} \Lambda r^{2}+\frac{1}{3} \Lambda a^{2}\left(1+\frac{2 M}{r}\right)
$$

to first order in the cosmological constant. Here $r_{*}$ is the unique positive root of $f(r)=0$ for sufficiently small $\Lambda$.

\section{Time delay}

Let us consider the time delay in the propagation of electromagnetic rays in the Kerr-de Sitter spacetime. The influence of the cosmological constant on the local propagation effects is expected to be very small; therefore, it is sufficient to consider the linearized Kerr-de Sitter metric in isotropic coordinates on the background Minkowski spacetime. In this case, the time that it takes for the rays to travel from the point $P_{1}:\left(t, \rho_{1}\right)$ to the point $P_{2}:\left(t, \rho_{2}\right)$ can be written as

$$
t_{2}-t_{1}=\left|\rho_{2}-\rho_{1}\right|+\Delta_{G E}+\Delta_{G M}+\Delta_{\Lambda},
$$

where $\rho$ is the position vector in the background Minkowski spacetime and $\Delta_{G E}$ and $\Delta_{G M}$ respectively represent the standard gravitoelectric and gravitomagnetic time delays [15]. Moreover, $\Delta_{\Lambda}$ is the additional time delay due to the cosmological constant. This delay can be calculated using the approach developed in [15]; that is,

$$
\Delta=\frac{1}{2 c} \int_{P_{1}}^{P_{2}} h_{\alpha \beta} k^{\alpha} k^{\beta} \mathrm{d} l
$$

where $h_{\alpha \beta}=g_{\alpha \beta}-\eta_{\alpha \beta}, k^{\alpha}=(1, \hat{\mathbf{k}}), \hat{\mathbf{k}}$ is a unit vector along the direction of propagation of the ray in the background Minkowski spactime and $\mathrm{d} l$ is the element of straight line connecting $P_{1}$ to $P_{2}$.

Concentrating on the cosmological constant, we note that for $M=a=0$, equation (1) reduces to the de Sitter metric. Under the coordinate transformation

$$
r(\rho)=\frac{\rho}{1+\frac{1}{12} \Lambda \rho^{2}},
$$

the de Sitter metric takes the isotropic form

$$
\mathrm{d} s^{2}=-\left(\frac{1-\psi}{1+\psi}\right)^{2} \mathrm{~d} t^{2}+\frac{1}{(1+\psi)^{2}}\left(\mathrm{~d} \rho^{2}+\rho^{2} \mathrm{~d} \Omega^{2}\right),
$$

where $\psi=\Lambda \rho^{2} / 12$. It follows from equation (16) that for the cosmological constant alone,

$$
h_{00}=\frac{1}{3} \Lambda \rho^{2}, \quad h_{0 i}=0, \quad h_{i j}=-\frac{1}{6} \Lambda \rho^{2} \delta_{i j} .
$$


Using these results in equation (14), we find

$$
\Delta_{\Lambda}=\frac{\Lambda}{12 c} \int_{P_{1}}^{P_{2}} \rho^{2} \mathrm{~d} l .
$$

It is straightforward to compute this integral and the result is

$$
\Delta_{\Lambda}=\frac{\Lambda}{36 c}\left|\rho_{\mathbf{1}}-\rho_{\mathbf{2}}\right|\left(\rho_{1}^{2}+\rho_{1} \cdot \rho_{\mathbf{2}}+\rho_{2}^{2}\right) .
$$

We note that for $\Lambda \approx 10^{-56} \mathrm{~cm}^{-2}, \Delta_{\Lambda} \sim 2 \mathrm{~s}$ for a ray of radiation crossing the disc of our galaxy.

The calculation of first-order gravitational delays in equation (13) assumes that the ray follows a straight line. Therefore, there must be in addition a geometrical delay that takes the actual deflected path into account; for weak lensing, the geometrical delay is of second order in the deflecting potentials. It is important to recognize that the cosmological constant does not participate in the deflection of light rays in the Kottler spacetime [16, 17]; therefore, the cosmological constant does not contribute to the geometrical delay in the Schwarzschild-de Sitter spacetime.

It follows from equation (19) that locally, i.e. for distances much smaller than $(3 M / \Lambda)^{1 / 3}$, the time delay due to the cosmological constant is very small compared to the corresponding Shapiro time delay. On the other hand, equation (19) indicates that for distances approaching $\Lambda^{-1 / 2}$, the time delay could be rather significant, but then our linear approximation scheme may break down. Thus for cosmological observations $\Delta_{\Lambda}$ should in general be taken into account, since the present uncertainty in the measurement of gravitational lensing time delay is about $\frac{1}{2}$ day [15].

\section{Orbital precession}

The solution of the equations of motion of a test particle in the Kerr-de Sitter spacetime is quite complicated. On the other hand, it turns out that one can obtain the main results regarding the gravitomagnetic clock effect, etc, from the linear approximation of general relativity. Therefore, we consider a linear post-Newtonian approach using a Lagrangian of the form $\mathcal{L}=-m \mathrm{~d} s / \mathrm{d} t$, where $m$ is the mass of the test particle and the metric is expressed in $(t, \rho)$ coordinates, where $\rho=(x, y, z)$ indicates a point in space with isotropic (post-Newtonian) Cartesian coordinates. The equations of motion then take the form

$$
\frac{\mathrm{d}^{2} \boldsymbol{\rho}}{\mathrm{d} t^{2}}+\frac{G M \rho}{\rho^{3}}=\mathbf{F},
$$

where $\mathbf{F}$ is the post-Newtonian perturbing function. The details of this process of reduction of the equations of motion to the form (20) are straightforward and have been described in detail in $[4,18]$. Under certain circumstances, the orbital perturbations can be easily characterized in terms of a modified orbit as has been done for the gravitomagnetic clock effect [4]. To illustrate this procedure, it is instructive to digress here and consider the case of bound motion in the Kerr-Taub-NUT spacetime; the results can be compared and contrasted with those of the Kerr-de Sitter spacetime. Linearizing this metric in the angular momentum and the NUT parameter of the source, we find the Schwarzschild metric together with the LenseThirring and Taub-NUT off-diagonal terms. Introducing the isotropic Schwarzschild radial coordinate $\rho$ and the corresponding Cartesian coordinates, we find that this gravitoelectric background is perturbed by a gravitomagnetic field,

$$
\mathbf{B}_{g}=\frac{G J}{c \rho^{5}}\left[3(\boldsymbol{\rho} \cdot \hat{\mathbf{J}}) \boldsymbol{\rho}-\rho^{2} \hat{\mathbf{J}}\right]-\frac{c^{2} \ell \boldsymbol{\rho}}{\rho^{3}}
$$


due to the angular momentum (i.e. the gravitomagnetic dipole moment $J$ ) and the gravitomagnetic monopole moment $\left(-c^{2} \ell\right)$ of the source. Here the NUT parameter $\ell$ has the dimension of length. The post-Newtonian gravitomagnetic orbital perturbation can then be obtained from equation (20), where $\mathbf{F}$ is of the form $\mathbf{F}=-2 \mathbf{v} \times \mathbf{B}_{\mathrm{g}} / c$. The linear perturbation of the orbit due to the angular momentum of the source has been worked out in detail in [4] in connection with the gravitomagnetic clock effect. Therefore, we only indicate here the orbital perturbations due to $\mathbf{F}_{\ell}=-2 c \ell \rho \times \mathbf{v} / \rho^{3}$ using the method developed in [4].

For the motion of a test mass around a gravitomagnetic monopole, the energy of the particle is conserved and so is its angular momentum, which is, however, augmented by a contribution from the interaction of the particle with the monopole, i.e. $\mathbf{j}=m \boldsymbol{\rho} \times \mathbf{v}-2 m c \ell \boldsymbol{\rho} / \rho$ is the conserved quantity. Following the analysis given in [4], let us note that the unperturbed orbit is given by a Keplerian ellipse in the $(X, Y)$-plane of the $(X, Y, Z)$ coordinate system that is related to the $(x, y, z)$ system by a rotation,

$$
\begin{aligned}
& x=\cos \Omega X-\sin \Omega \cos i Y+\sin \Omega \sin i Z, \\
& y=\sin \Omega X+\cos \Omega \cos i Y-\cos \Omega \sin i Z, \\
& z=\sin i Y+\cos i Z,
\end{aligned}
$$

where $i$ is the inclination angle and $\Omega$ is the longitude of the line of the ascending nodes. The Keplerian ellipse is then given by $X=\rho \cos \varphi, Y=\rho \sin \varphi$ and $Z=0$, where

$$
\rho=\frac{a\left(1-e^{2}\right)}{1+e \cos (\varphi-g)}, \quad \frac{\mathrm{d} \varphi}{\mathrm{d} t}=\frac{L_{0}}{\rho^{2}}
$$

and $L_{0}=\sqrt{G M a\left(1-e^{2}\right)}$ is the specific orbital angular momentum of the unperturbed orbit. Here $a, e$ and $g$ are respectively the semimajor axis, eccentricity and argument of the pericentre of the unperturbed elliptical orbit.

Once the gravitomagnetic monopole moment of the source is turned on at $t=0$, when observations begin, the only change that occurs in the orbit to linear order is that $Z$ is no longer zero but is given by

$$
Z=\frac{2}{c} \mu L_{0} \frac{1-\cos \left(\varphi-\varphi_{0}\right)}{1+e \cos (\varphi-g)}
$$

where $\mu=-c^{2} \ell /(G M)$ is the dimensionless strength of the monopole and $\varphi=\varphi_{0}$ at $t=0$. A detailed examination shows that there is no gravitomagnetic clock effect in this case to first order in $\ell$. In this linear order, the net angular momentum $\mathbf{j}$ turns out to have components $j_{X}=-2 m c \ell \cos \varphi_{0}, j_{Y}=-2 m c \ell \sin \varphi_{0}$ and $j_{Z}=m L_{0}$.

The gravitomagnetic monopole moment of the source presumably exists for all time; therefore, the procedure described above has to be reinterpreted in terms of an osculating ellipse. Namely, the position and velocity of the test mass at any given instant of time define an elliptical orbit that is momentarily tangent to the actual perturbed orbit. Therefore, the perturbed motion may be described in terms of the evolution of the osculating ellipse as in the Lagrange planetary equations. In the case under consideration here the observations are assumed to begin at $t=0$; hence, the elliptical orbit (25) is simply the osculating ellipse of the perturbed orbit at $t=0$.

Let us now return to the Kerr-de Sitter metric and linearize it in the small parameters $\epsilon=a / M$ and $\delta=\Lambda M^{2}$ with $M \neq 0$. We then need to introduce an isotropic radial coordinate $\rho$ that puts the Kottler metric in isotropic form. The connection between $r$ and $\rho$ is given by the differential equation

$$
\left(\frac{\mathrm{d} r}{\mathrm{~d} \rho}\right)^{2}=\frac{r^{2}}{\rho^{2}}\left(1-\frac{2 M}{r}-\frac{\Lambda}{3} r^{2}\right) .
$$


To first order in $\delta$, we find that

$$
r(\rho)=\rho\left(1+\frac{M}{2 \rho}\right)^{2}-\frac{\delta}{24} \rho\left(1-\frac{M^{2}}{4 \rho^{2}}\right) I\left(\frac{2 \rho}{M}\right),
$$

where $I(x)$ is given by

$$
I(x)=\int^{x} \frac{(1+u)^{6}}{(1-u)^{2}} \frac{\mathrm{d} u}{u^{3}} .
$$

This integral can be evaluated and the result is

$$
2 I(x)=K+x^{2}+16 x+60 \ln x-\frac{128}{x-1}-\frac{16}{x}-\frac{1}{x^{2}},
$$

where $K$ is a dimensionless integration constant that we can set equal to zero for the sake of simplicity. A detailed examination of the resulting equation of motion then shows that the dominant effect of the cosmological constant would simply be due to the effective Newtonian perturbation of the form $\mathbf{F}=\lambda \rho$, where $\lambda=\Lambda c^{2} / 3$. Under such a perturbation the orbital angular momentum is conserved and the orbit is thus planar. On the other hand, the orbital energy is augmented by a contribution from the cosmological constant so that the conserved quantity is $E$, where $2 E=v^{2}-\lambda \rho^{2}-2 G M / \rho$. Though the perturbing function is simply proportional to $\rho$ in this case, it turns out that the simple method developed in [4] is not directly applicable in this case for orbits of arbitrary eccentricity since the perturbing function has the form of an infinite series in powers of the eccentricity (cf section 5). This is in contrast with the Kerr-Taub-NUT case. It is therefore necessary to adopt a different approach to the motion of the perturbed orbit unless $e \ll 1$, in which case the method of [4] is adequate. The general solution to first order in the cosmological constant is considered in the next section; in the following, we study the average motion of the perturbed orbit.

It is interesting to compute the average rate of precession of the pericentre under the influence of $\Lambda$. To this end, let us introduce the Runge-Lenz vector of the perturbed orbit

$$
\mathbf{Q}=\mathbf{v} \times \mathbf{L}-G M \rho / \rho,
$$

where $\mathbf{L}=L_{0} \hat{\mathbf{Z}}$ and we note that for $\mathbf{F}=0, \mathbf{Q}_{\mathbf{0}}=G M e(\cos g, \sin g, 0)$ in the $(X, Y, Z)$ coordinate system. For $\mathbf{F}=\lambda \rho$, the orbit remains in the $(X, Y)$-plane and $\dot{Q}_{X}=\lambda L_{0} Y, \dot{Q}_{Y}=$ $-\lambda L_{0} X$ and $\dot{Q}_{Z}=0$. Thus in this case

$$
\frac{\mathrm{d} \mathbf{Q}}{\mathrm{d} t}=-\lambda \mathbf{L} \times \boldsymbol{\rho} .
$$

Averaging this relation over the 'fast' motion, i.e. the unperturbed orbital motion of the test particle (with period $T$ ) would reveal the 'slow' (secular) behaviour of the Runge-Lenz vector over a long period of time. Defining the average over a period as

$$
\langle f\rangle=\frac{1}{T} \int_{0}^{T} f \mathrm{~d} t=\left(1-e^{2}\right)^{3 / 2} \frac{1}{2 \pi} \int_{0}^{2 \pi} \frac{f(\varphi) \mathrm{d} \varphi}{[1+e \cos (\varphi-g)]^{2}},
$$

we obtain

$$
\langle X\rangle=-\frac{3}{2} a e \cos g, \quad\langle Y\rangle=-\frac{3}{2} a e \sin g,
$$

where we have used the relation

$$
\frac{1}{2 \pi} \int_{\zeta_{0}}^{\zeta_{0}+2 \pi} \frac{\cos \zeta \mathrm{d} \zeta}{(1+e \cos \zeta)^{3}}=-\frac{3}{2} \frac{e}{\left(1-e^{2}\right)^{5 / 2}}
$$

Thus we find that

$$
\left\langle\frac{\mathrm{d} \mathbf{Q}}{\mathrm{d} t}\right\rangle=\Omega \times \mathbf{Q}_{\mathbf{0}}
$$


where

$$
\Omega=\frac{3}{2} \frac{a \lambda L_{0}}{G M} \hat{\mathbf{Z}}=\frac{1}{2} \frac{\Lambda c^{2}}{\omega} \sqrt{1-e^{2}} \hat{\mathbf{Z}} .
$$

Here $\omega=2 \pi / T$ is the Keplerian frequency of the unperturbed orbit. For $\Lambda \approx 10^{-56} \mathrm{~cm}^{-2}$, the precession frequency (37) is too small to be observable at present in the solar system [16]. The precession angle after one period is given by

$$
\frac{\pi c^{2} \Lambda a^{3}}{G M} \sqrt{1-e^{2}}
$$

To first order in eccentricity, this result agrees with previous work [16, 19], while the exact dependence on eccentricity as $\sqrt{1-e^{2}}$ differs from $\left(1-e^{2}\right)^{3}$ given in [19].

To gain a deeper understanding of the average behaviour of the orbit, we consider the osculating ellipse in this case and note that the orbital elements of this ellipse vary with time according to the Lagrange planetary equations [20] as

$$
\begin{aligned}
\frac{\mathrm{d} a}{\mathrm{~d} t} & =\frac{2 \lambda a e \sqrt{1-e^{2}}}{\omega} \frac{\sin (\varphi-g)}{1+e \cos (\varphi-g)}, \\
\frac{\mathrm{d} e}{\mathrm{~d} t} & =\frac{1-e^{2}}{2 e a} \frac{\mathrm{d} a}{\mathrm{~d} t}, \\
\frac{\mathrm{d} g}{\mathrm{~d} t} & =-\frac{\lambda \sqrt{\left(1-e^{2}\right)^{3}}}{e \omega} \frac{\cos (\varphi-g)}{1+e \cos (\varphi-g)} .
\end{aligned}
$$

Employing the averaging method once again, we find that

$$
\begin{aligned}
& \left\langle\frac{\mathrm{d} a}{\mathrm{~d} t}\right\rangle=0, \quad\left\langle\frac{\mathrm{d} e}{\mathrm{~d} t}\right\rangle=0, \\
& \left\langle\frac{\mathrm{d} g}{\mathrm{~d} t}\right\rangle=\frac{3}{2} \frac{\lambda}{\omega} \sqrt{1-e^{2}},
\end{aligned}
$$

where relation (35) has been used. The rate of advance of the pericentre given by equation (43) is consistent with our previous result. Thus in the presence of a cosmological constant the orbital plane does not change and the orbit keeps its shape on the average while precessing with frequency $\Lambda c^{2} \sqrt{1-e^{2}} /(2 \omega)$.

It follows from equation (37) that the frequency of the pericentre precession caused by the cosmological constant is directly proportional to the period of the binary orbit, which must therefore be very large for the effect to be perceptible. The ratio of this precession frequency to the Einstein pericentre precession frequency is given by $\Lambda a^{4} c^{4}\left(1-e^{2}\right)^{3 / 2} /\left(6 G^{2} M^{2}\right)$, which is $\sim 10^{-14}$ for the motion of the Earth around the Sun. We conclude that the influence of the cosmological constant on orbital motion is too small to be measurable in the solar system.

\section{Perturbed Keplerian motion}

It is interesting to give the general solution of equation (20) for $\mathbf{F}=\lambda \rho$ to first order in the cosmological constant. For a positive cosmological constant, the effective force on the test mass is along the radial direction away from the source. The orbit is thus planar and $\rho^{2} \mathrm{~d} \varphi / \mathrm{d} t=L_{0}$ is the constant specific orbital angular momentum. In terms of $u=\rho^{-1}$, the radial part of equation (20) reduces in this case to

$$
\frac{\mathrm{d}^{2} u}{\mathrm{~d} \varphi^{2}}+u=p^{-1}-\frac{\lambda}{L_{0}^{2} u^{3}},
$$


where $p=L_{0}^{2} /(G M)$ is a positive constant. The right-hand side of equation (44) can be expressed as a function of $\varphi$ if we substitute for $u$ the unperturbed solution in accordance with our linear perturbation scheme. The unperturbed solution is given by

$$
u_{0}=\frac{1}{p}(1+e \cos \varphi)
$$

where we have chosen the planar Cartesian coordinates such that $g=0$ for the sake of simplicity. Here $e \geqslant 0$ is the orbital eccentricity of the conic section. For an ellipse $e<1$ and $p=a\left(1-e^{2}\right)$, where the pericentre distance from the focus is $\rho_{\min }=a(1-e)$ and $a$ is the semimajor axis. For a parabola $e=1$ and $\rho_{\min }=p / 2$ is the pericentre distance, while for a hyperbola $e>1$ and $p=a\left(e^{2}-1\right)$, where the pericentre distance from the focus is $\rho_{\min }=a(e-1)$. Substituting equation (45) for $u$ in the right-hand side of equation (44), the resulting equation can be transformed to the form

$$
\left(1-\xi^{2}\right) \frac{\mathrm{d}^{2} U}{\mathrm{~d} \xi^{2}}-\xi \frac{\mathrm{d} U}{\mathrm{~d} \xi}+U=\frac{q}{(1+e \xi)^{3}},
$$

where $\xi=\cos \varphi, U=u-p^{-1}$ and $q=-\lambda p^{3} / L_{0}^{2}$. Equation (46) can be integrated once and we find

$$
\left(1-\xi^{2}\right) \frac{\mathrm{d} U}{\mathrm{~d} \xi}+\xi U=-\frac{q}{2 e(1+e \xi)^{2}}+C
$$

where $C$ is an integration constant. Equation (47) can be written in the form

$$
\frac{\mathrm{d}}{\mathrm{d} \xi}\left[\left(1-\xi^{2}\right)^{-1 / 2} U\right]=-\frac{q}{2 e\left(1-\xi^{2}\right)^{3 / 2}(1+e \xi)^{2}}+\frac{C}{\left(1-\xi^{2}\right)^{3 / 2}}
$$

and integrated using formulae (2.264), (2.266) and (2.268) given in [21]. The result for an elliptical orbit is

$U=\frac{1}{2} \frac{q}{\left(1-e^{2}\right)^{2}}\left[3(1+e \mathcal{I} \sin \varphi)-\frac{1-e^{2}}{1+e \cos \varphi}-\left(2 e+\frac{1}{e}\right) \cos \varphi\right]+C \cos \varphi+S \sin \varphi$,

where $S$ is a constant of integration and

$$
\mathcal{I}=\frac{1}{\sqrt{1-e^{2}}} \arcsin \left(\frac{e+\cos \varphi}{1+e \cos \varphi}\right) .
$$

The integration of equation (48) can be similarly carried out for parabolic and hyperbolic orbits using the relevant formulae of [20]. We expect that $u$ reduces to the unperturbed solution $u_{0}$ as $\lambda \rightarrow 0$; therefore, it is useful to set $C=q C^{\prime}+e / p$ and $S=q S^{\prime}$. The constants $C^{\prime}$ and $S^{\prime}$ can be uniquely determined using the initial conditions, i.e. the initial position and velocity of the test mass. In this way, we find the orbital radius $\rho=u^{-1}=\left(p^{-1}+U\right)^{-1}$ and then $\varphi(t)$ by integrating $\mathrm{d} \varphi / \mathrm{d} t=L_{0} \rho^{-2}$.

\section{Discussion}

In this paper we have considered some of the local physical consequences of the existence of a cosmological constant. In particular, we have considered the motion of test bodies and the propagation of rays in the Kerr-de Sitter spacetime. While the clock effect is rather weakly affected by the presence of a cosmological constant, there is a (gravitoelectric) time delay in the propagation of rays directly proportional to $\Lambda$. Though this effect is too small to be measurable in the solar system, it should in general be taken into account in the interpretation of gravitational lensing time delay. Moreover, the average behaviour of bounded orbits has 
been examined and the general rate of orbital precession has been determined for an arbitrary eccentric orbit. On the average, the orbital plane remains invariant and the orbit keeps its shape but precesses due to the presence of a cosmological constant; however, the precession rate is too small to be detectable in the solar system.

\section{References}

[1] Cohen J M and Mashhoon B 1993 Phys. Lett. A 181353

[2] Mashhoon B, Gronwald F and Theiss D S 1999 Ann. Phys., Lpz 8135

[3] Mashhoon B, Gronwald F and Lichtenegger H I M 2000 Gyros, Clocks, Interferometers .... : Testing Relativistic Gravity in Space ed C Lämmerzahl, C W F Everitt and F W Hehl (Berlin: Springer) pp 83-108

[4] Mashhoon B, Iorio L and Lichtenegger H 2001 Phys. Lett. A 29249

[5] Demiański M 1973 Acta Astron. 23197

[6] Carter B 1973 Black Holes ed C DeWitt and B S DeWitt (New York: Gordon and Breach) pp 101-3

[7] Kottler F 1918 Ann. Phys., Lpz $\mathbf{5 6} 410$

[8] Schmidt H-J 1993 Fortschr. Phys. 41179

[9] Liu H and Mashhoon B 1995 Ann. Phys., Lpz 4565

[10] Peebles P J E and Ratra B 2003 Rev. Mod. Phys. 75 (Preprint astro-ph/0207347) 559-606

[11] Howes R J 1981 Gen. Rel. Grav. 13829

[12] Bini D, Jantzen R T and Mashhoon B 2001 Class. Quantum Grav. 18653

[13] Bini D, Jantzen R T and Mashhoon B 2002 Class. Quantum Grav. 1917

[14] Maartens R, Mashhoon B and Matravers D R 2002 Class. Quantum Grav. 19195

[15] Ciufolini I, Kopeikin S, Mashhoon B and Ricci F 2003 Phys. Lett. A 308101

[16] Islam J N 1983 Phys. Lett. A 97239

[17] Lake K 2002 Phys. Rev. D 65087301

[18] Mashhoon B 2001 Reference Frames and Gravitomagnetism ed J-F Pascual-Sánchez, L Floría, A San Miguel and F Vicente (Singapore: World Scientific) pp 121-32

[19] Rindler W 2001 Relativity: Special, General, and Cosmological (Oxford: Oxford University Press) p 305

[20] Danby J M A 1988 Fundamentals of Celestial Mechanics 2nd edn (Richmond: Willmann-Bell)

[21] Gradshteyn I S and Rhyzhik M 1980 Table of Integrals, Series and Products (New York: Academic) 